\title{
Higher Selective Ion Transportation Using Functional Metal Coated PTFE Membrane with Electrical Bias
}

\author{
Jeong Hwan Kim ${ }^{1,2}$, Seung-Hun Lee ${ }^{1}$, Sang-Hoon $\mathbf{J o}^{1}$, Seungmin Hyun ${ }^{1,2}$, Kwanoh Kim ${ }^{1}$, Doo-Sun Choi ${ }^{1,2}$, \\ Jae Sung Yoon ${ }^{1,2}$, Yeong-Eun Yoo, \\ ${ }^{1}$ Department of Nano Manufacturing Technology, Korea Institute of Machinery and Materials (KIMM) \\ Gajeongbuk-ro 156, Daejeon, South Korea \\ ${ }^{2}$ Department of Nano-Mechatronics, University of Science and Technology \\ Gajeong-ro 217, Daejeon, South Korea \\ jkim@kimm.re.kr; jkimatust@ust.ac.kr
}

\section{Extended Abstract}

Functional polymer membrane has been highlighted due to its higher functionality in separation process for liquid and gaseous mixtures (gas separation, reverse osmosis, nano/micro/ultra-filtrations) and in other important applications of membranes, such as bio-materials, catalysis production. Especially, artificial bio-membrane system (e.g. artificial kidney or artificial aquaporin) has been intensively researched to develop the water molecule transportation system based on artificial nanoscale channel system. [1,2] Unlike micro-sized particles, the selective transportation of ions is difficult in water, because the dissolved ions are very small. However, the control of ions and molecules transportation in water is very significant in order to develop artificial bio-membrane system. Therefore, we investigated the selective ion transportation in $\mathrm{NaNO}_{3}$ solution by using functional polymer membrane including conductive metal layer and applied electrical bias on it.

In this study, sputtered Platinum (Pt) film was coated on Polytetrafluoroethylene (PTFE) membrane which has higher chemical resistance, in order to form conductive layer on PTFE membrane surface. The Titanium (Ti) film was inserted for improved adhesion between Pt film and PTFE membrane. The ground electrode was located in the $\mathrm{NaNO}_{3}$ solution. The flow of $\mathrm{NaNO}_{3}$ solution was precisely controlled by using the syringe pump (Legato 200). In order to apply constant electrical voltage to the Pt layer on PTFE membrane, Keithley 2400 sourcemeter was used with measuring the current and resistance between Pt surface on PTFE membrane and ground electrode. After generating an electric field by applying various voltages $( \pm 150 \mathrm{~V}, \pm 100 \mathrm{~V}, \pm 50 \mathrm{~V})$ between the ground electrode and the Pt layer on the PTFE membrane, and the $\mathrm{NaNO}_{3}$ solution was passed through the Pt coated PTFE membrane sample at a constant flow rate $(0.5 \mathrm{ml} / \mathrm{min})$. The concentration of $\mathrm{NaNO}_{3}$ was $100 \mathrm{ppm}$ and the filtering of ions was performed twice at each applied electrical voltage. Lastly, ion concentration of solution which passed through the Pt film coated membrane was measured using a nitrate ion selective electrode (C Mettler Toledo Inc.). In addition, ALD (Atomic layer deposition) process was conducted in order to investigate the effect of the surface functionalization on PTFE surface.

The ion filtering efficiency and the voltage polarity and level of voltage dependence on ions transportation was studied. As the electrical voltage is applied across the channel, selective ions are rapidly enriched or depleted at the electrode applied positive or negative voltage. Under negative electrical bias $(-150 \mathrm{~V})$, the Pt film coated PTFE membrane system showed above $20 \%$ reduction of nitrate ions in $\mathrm{NaNO}_{3}$ solution. By filtering twice using Pt film coated PTFE membrane under negative electrical bias, $38 \%$ of nitrate ions was reduced in $\mathrm{NaNO}_{3}$ solution. Under positive electrical voltage $(+150 \mathrm{~V})$, the sodium ion was effectively reduced by $20 \%$ approximately.

We showed that selective ions through PTFE-membrane connected to a voltage source can be efficiently transported and controlled by applying functional metal layer on PTFE-membrane. In addition, solution including $\mathrm{NaCl}$ was also investigated in this study. Selective ion transportation through Pt film coated PTFE membrane with electrical bias will be systemically presented using proposed mechanism and experimental results. 


\section{References}

[1] V. O. Osipenko, M. N. Balakina, D. D. Kucheruk, and V. V. Goncharuk, "Water Purification of Nitrates with Their Deep Concentration by the Method of Electrodialysis," J. Water Chem. and Tech., vol. 36, no. 2, pp. 75-79, 2014.

[2] X. Zhu, W. He, B. E. Logan, "Influence of solution concentration and salt types on the performance of reverse electrodialysis cells,” J. Membrane Science, vol. 494, pp. 154-160, 2015. 